Published in final edited form as:

Schizophr Res. 2017 November ; 189: 169-174. doi:10.1016/j.schres.2017.02.014.

\title{
Ventricular enlargement and progressive reduction of cortical gray matter are linked in prodromal youth who develop psychosis
}

\author{
Yoonho Chung, MS ${ }^{a}$, Kristen M. Haut, PhD ${ }^{b}$, George He, PhDa , Theo G.M. van Erp, PhD', \\ Sarah McEwen, $\mathrm{PhD}^{\mathrm{d}}$, Jean Addington, $\mathrm{PhD}^{\mathrm{e}}$, Carrie E. Bearden, $\mathrm{PhD}^{\mathrm{d}}$, Kristin Cadenhead, \\ MD $^{f}$, Barbara Cornblatt, PhDg, Daniel H. Mathalon, PhD, MD ${ }^{h}$, Thomas McGlashan, MDi, \\ Diana Perkins, MDj,k, Larry J. Seidman, $\mathrm{PhD}^{\mathrm{j}}{ }^{\mathrm{m}}$, Ming Tsuang, MD, $\mathrm{PhD}^{\mathrm{f}}$, Elaine Walker, \\ PhD $^{n}$, Scott W. Woods, MD ${ }^{i}$, and Tyrone D. Cannon, PhD ${ }^{a, i}$ on behalf of the North American \\ Prodrome Longitudinal Study (NAPLS) Consortium* \\ aDepartment of Psychology, Yale University, 2 Hillhouse Ave., New Haven, CT 06520-8205, \\ United States \\ ${ }^{b}$ Department of Psychiatry, Rush University Medical Center., 1750 W Harrison St, Jelke Ste 1425, \\ Chicago, IL 60612 \\ 'Department of Psychiatry and Human Behavior, UC Irvine, 101 The City Drive, Irvine, CA, \\ 92697, United States
}

'Semel Institute for Neuroscience and Human Behavior and Department of Psychology, UCLA, 760 Westwood Plaza, Los Angeles, CA, 90095, United States

eDepartment of Psychiatry, University of Calgary, 3280 Hospital Drive NW, Calgary, AB T2N4Z6, Canada

fDepartment of Psychiatry, UCSD, 9500 Gilman Drive, La Jolla, CA 92093-0761, United States

9Department of Psychiatry, Zucker Hillside Hospital, 75-59 263rd St., Queens, NY 11004, United States

hDepartment of Psychiatry, UCSF, 401 Parnassus Avenue, San Francisco, CA 94143, United States

'Department of Psychiatry, Yale University, 300 George St., New Haven, CT 06511 United States jDepartment of Psychiatry, University of North Carolina, Chapel Hill, 101 Manning Dr, Chapel Hill, NC 27514, United States

Address Correspondence: Tyrone D. Cannon, PhD, Department of Psychology, 2 Hillhouse Avenue, New Haven, CT 06511, tyrone.cannon@yale.edu, Phone: 203-436-1545, Fax: 203-432-7172.

Conflict of Interest

The authors have declared that there are no conflicts of interest in relation to the subject of this study. Dr. Cannon reports that he is a consultant to the Los Angeles County Department of Mental Health and to Boerhinger Ingelheim Pharmaceutical and is a co-inventor (with the other NAPLS investigators) on a pending patent of a blood-based predictive biomarker for psychosis..

Publisher's Disclaimer: This is a PDF file of an unedited manuscript that has been accepted for publication. As a service to our customers we are providing this early version of the manuscript. The manuscript will undergo copyediting, typesetting, and review of the resulting proof before it is published in its final citable form. Please note that during the production process errors may be discovered which could affect the content, and all legal disclaimers that apply to the journal pertain. 


\author{
kRenaissance Computing Institute, University of North Carolina, Chapel Hill \\ 'Department of Psychiatry, Beth Israel Deaconess Medical Center and Harvard Medical School, \\ 401 Park Drive, 2 East, Boston, MA 02215, United States \\ mDepartment of Psychiatry, Massachusetts General Hospital and Harvard Medical School, 401 \\ Park Drive, 2 West, Boston, MA 02215, United States \\ nDepartment of Psychology, Emory University, 487 Psychology Building, 36 Eagle Row, Atlanta, \\ GA 30322, United States
}

\title{
Abstract
}

In a recent prospective longitudinal neuroimaging study, clinical high-risk (CHR) individuals who later developed full-blown psychosis showed an accelerated rate of gray matter thinning in superior and medial prefrontal cortex (PFC) and expansion of the ventricular system after applying a stringent correction for multiple comparisons. Although cortical and subcortical volume loss and enlarged ventricles are well characterized structural brain abnormalities among patients with schizophrenia, no prior study has evaluated whether these progressive changes of neuroanatomical indicators are linked in time prior to onset of psychosis. Therefore, we investigated the relationship between the changes in cortical gray matter thickness and ventricular volume using the longitudinal neuroimaging data from the North American Prodrome Longitudinal Study at the whole-brain level. The results showed that ventricular expansion is linked in time to progressive reduction of gray matter, rather than to structural changes in proximal subcortical regions, in a broadly distributed set of cortical regions among CHR youth, including superior, medial, lateral, and inferior PFC, superior temporal gyrus, and parietal cortices. In contrast, healthy controls did not show the same pattern of associations. The main findings were further replicated using a third assessment wave of MRI scans in a subset of study participants who were followed for an additional year. These findings suggest that the gray matter regions exhibiting aberrant rates of thinning in relation to psychosis risk are not limited to the PFC regions that survived the statistical threshold in our primary study, but also extend to other cortical regions previously implicated in schizophrenia.

\section{Keywords}

Schizophrenia; psychosis; prodromal; MRI; Ventricle; CHR

\section{Introduction}

A recent prospective longitudinal neuroimaging study of individuals at clinical high-risk (CHR) for psychosis revealed that those who converted to psychosis showed accelerated gray matter reduction in superior and medial prefrontal cortex (PFC) and expansion of the ventricular system compared with CHR subjects who did not convert and healthy controls (Cannon et al., 2015). These aberrant brain trajectories were predicted by initial prodromal symptom severity (Chung et al., 2015) and were not explained by exposure to antipsychotics (Cannon et al., 2015). However, two interrelated questions remain to be resolved: 1) Is the accelerated gray matter decline during the psychosis prodrome specific to the regions of 
PFC that survived vertex-wise correction for multiple comparisons?; and 2) Is ventricular expansion a consequence of this cortical thinning or of more proximal (i.e., subcortical) tissue loss?

As prefrontal cortical regions continue to mature via synaptic pruning and myelination throughout adolescence (Gogtay et al., 2004; Huttenlocher, 1979; Huttenlocher and Dabholkar, 1997), an accelerated rate of gray matter loss in this region observed during the psychosis prodrome may explain why onset of schizophrenia and related disorders commonly occurs during late adolescence (Cannon et al., 2015). If over-pruning is the mechanism for accelerated gray matter reduction, then the greatest rate of synaptic loss should be occurring in regions that are undergoing maximal pruning at the time. However, given that the pruning process occurs throughout the cortex to some degree during adolescence, the acceleration effects associated with onset of psychosis may be distributed more broadly.

Neuroimaging studies such as those referred to above rely on mass-univariate statistics to identify regions that show differential change linked to onset of psychosis. Given that tens of thousands of statistical comparisons are conducted (e.g., on each vertex in the cortical surface and each subcortical region), adjustment of the observed probabilities (e.g., by means of the False Discovery Rate or similar procedure) is required to guard against type I error. Although this approach is an effective strategy in elucidating brain areas that are likely to be affected by disease processes, it has been also criticized for biasing results toward highly localized regions that survive the statistical threshold employed (Davatzikos, 2004). This may help to explain why cortical regions that are generally reduced in thickness among patients with schizophrenia, including superior temporal gyrus, parietal cortex, and lateral and inferior aspects of prefrontal cortex, were not among those detected as showing a significantly steeper rate of decline among converters (Cannon et al., 2015). It is notable in this regard that these regions were associated with significantly differential reductions among converters when not correcting for multiple comparisons.

Previous studies have shown that cortical gray matter volumes are inversely related with ventricular volumes among patients with schizophrenia (DeLisi et al., 2004; 1997; Gaser et al., 2004; Symonds et al., 1999). However, no prior study has evaluated whether progressive changes in these two anatomical indicators are linked in time prior to onset of psychosis. Ventricular expansion could be a consequence of thinning of the cortical gray matter ribbon in regions that undergo accelerated loss in association with onset of psychosis, or it could reflect tissue loss in more proximal brain structures such as thalamus, basal ganglia, and hippocampus (Gaser et al., 2004). One reason to suspect that the ventricular changes are linked with cortical, rather than subcortical, reductions in gray matter prior to psychosis onset is that the subcortical structures do not appear to show differential change among CHR converters to psychosis (Cannon et al., 2015). If this interpretation is correct, the explanation would likely involve reductions in extra-ventricular mechanical pressures against the ventricular walls as the cortical ribbon thins (rather than CSF expanding to "invade" areas of adjacent tissue loss). 
Based on the foregoing, we hypothesized that the rate of expansion of the ventricular system would be highly linked in time with the rate of cortical gray matter reduction among CHR patients. Although we expected this relationship to be maximal in superior and medial aspects of PFC (where converters showed the greatest differential reductions), we hypothesized that it would also be present in other cortical regions in which patients with schizophrenia have gray matter deficits, including lateral and inferior PFC, superior temporal gyrus, and parietal cortex (Cannon et al., 2015; Pantelis et al., 2003; Rimol et al., 2012; Shenton et al., 2001).

\section{Methods}

\subsection{Participants}

The study protocol and consent form was reviewed and approved by the Institutional Review Boards at each of the 8 data collection sites (UCLA, Emory, Beth Israel Deaconess Medical Center, Zucker Hillside Hospital, UNC, UCSD, Calgary, Yale). Participants were evaluated using the Structured Interview for Prodromal Syndromes (SIPS) (McGlashan et al., 2010) and the Structured Clinical Interview for Axis I DSM-IV Disorders (Addington et al., 2012; First et al., 1995). CHR cases were defined as those who met SIPS/SOPS criteria for a psychosis risk syndrome (McGlashan et al., 2010) excluding cases who had ever met DSMIV criteria for a psychotic disorder. Summary of demographics are shown in Table 1 and in prior studies (Cannon et al., 2015; Chung et al., 2015)

\subsection{MRI Scans and Image Processing}

Detailed information about MRI protocol, image processing, and test-retest reliability are described in previous studies (Cannon et al., 2015; 2013). Briefly, five sites operated Siemens scanners and three sites operated GE scanners, all at 3 Tesla; optimized for each scanner manufacturer, software version and coil configuration according to the ADNI protocol (http://adni.loni.ucla.edu/research/protocols/mri-protocols/). MRI structural data were acquired at baseline (BL) and 12-month follow-up (12-FU), and follow-up scans for those who developed fully psychotic symptoms were assessed at the point of conversion.

Surface-based cortical reconstruction, estimation of cortical thickness and subcortical volumetric segmentation was performed using the automated Freesurfer pipeline (http:// surfer.nmr.mgh.harvard.edu/) (Dale et al., 1999; Fischl and Dale, 2000; Fischl et al., 2002; 1999; 2004). The reconstructed baseline and follow-up scans were further processed using Freesurfer's longitudinal stream (Reuter and Fischl, 2011; Reuter et al., 2010; 2012).

\subsection{Statistical Analysis}

Imaging measures were first transformed to annualized rates of percent change (ARCH) in each cortical vertex and subcortical region of interest $(\mathrm{ROI})$, where $\mathrm{ROI}_{\mathrm{ARCH}}=\left(\left(\mathrm{ROI}_{\mathrm{FU}}-\right.\right.$ $\mathrm{ROI}_{\mathrm{BL}}$ )/ROI $\mathrm{RL}_{\mathrm{BL}}$ /Interval (years). Relationships between $\mathrm{ARCH}$ of total ventricle volume (i.e., sum of lateral and third ventricle volume) and ARCH of cortical gray matter values were tested vertex-wise using the general linear model. False Discovery Rate (FDR) correction was set to $1 \%$ (Genovese et al., 2002). Age, sex, and scanner were treated as nuisance covariates. 
For additional ROI-based statistical tests, ARCH was calculated in particular cortical and subcortical ROIs using Freesurfer (Desikan et al., 2006). In particular, superior frontal cortex (SFC) from the Desikan atlas was chosen as an exemplary ROI as this region showed accelerated rate of gray matter decline among converters exhibiting high level of unusual thought content in our previous studies (Cannon et al., 2015; Chung et al., 2015). For all ROI-based analyses, Bonferroni correction was applied when appropriate. To protect correlation coefficients from being skewed by outliers, all analyses were run with statistical outliers excluded. An outlier was defined when Studentized deleted residuals exceeded + -2.5 .

For the purpose of testing the replicability of our main hypotheses, subset of CHR and control subjects were followed for an additional third time point for a 24-month (24-FU) MRI assessment. Parallel set of ROI-based statistical tests were performed as described above.

\section{Results}

\subsection{Cross-sectional analyses at baseline}

There were no significant differences in the relationship between ventricular volume and cortical thickness between converters, non-converters, and healthy controls cross-sectionally at baseline.

\subsection{Longitudinal expansion of ventricular volume and cortical reduction}

FreeSurfer-based cortical surface analysis was used to investigate the spatial relationship between longitudinal changes in ventricular volume and cortical gray matter thickness using GLM. As shown in Figure 1, a significant inverse relationship was observed among CHR individuals, where higher rates of ventricular expansion were associated with steeper rates of gray matter thinning across the cortical surface, which are represented in the Figure by the cooler colors. Strong negative relationships were evident bilaterally in superior, lateral, and medial prefrontal, parietal, and superior temporal cortices. To characterize the strength of the relationships, partial correlation coefficients (r) were also mapped in Figure 1. Similarly to the thresholded P-value maps, regions in medial and lateral prefrontal, parietal and temporal cortices in both right and left hemispheres showed relatively robust effect sizes that were larger than in the remaining cortical areas. The same vertex-wise analysis was done on matched healthy controls, but only sparse regions showed significant associations (see Supplementary Figure 1 for statistical maps). Vertex-wise statistical maps focusing specifically on cortical regions correlated with third ventricle expansion are provided in Supplementary Figure 2.

Next, we tested for statistical differences in the relationship of cortical thinning and ventricular expansion by clinical group (i.e., CHR vs healthy controls) and included ARCH ventricular volume by the group interaction term in the model. We selected the mean of left and right SFC parcel (Desikan et al., 2006) as a dependent measure, based on our previous findings mentioned above (Cannon et al., 2015; Chung et al., 2015) and as this parcel nicely circumscribes clusters of significant regions with highest effect sizes revealed from the 
vertex-wise analysis in this study (Figure 1). The tested regression model $(F[5,389]=7.98$, $p<0.0001$ ) showed a significant interaction effect (ARCH ventricular volume $\mathrm{x}$ group interaction term: $t=2.3, p=0.032$ ), in which adjusting for age and sex, CHR cases with higher rates of ventricular expansion showed steeper rates of gray matter thinning in the SFC region $(r=-3.5, p<0.0001, \mathrm{df}=261)$, whereas healthy controls revealed no relationship $(r$ $=-0.1, p=0.25, \mathrm{df}=128$ ). See Supplementary Figure 3 for scatterplot.

We further partitioned the CHR sample into converters and non-converters and and reran the model above. In this model $(\mathrm{F}[7,387]=8.11, p<0.0001)$, the slope of ventricular volume change among converters was statistically significantly different from that of healthy controls $(t=-2.82, p=0.0049)$. However, the interaction term for non-converters was not significantly different from healthy controls $(t=-1.39, p<0.16)$. Among converters, partial correlation analysis revealed a strong negative correlation, where expansion of ventricular volume was significantly associated with steeper rate of SFC thinning $(r=-0.51, p<0.001$, $\mathrm{df}=33)$. This association was also significant among non-converters $(r=-0.24, p<0.001$, $\mathrm{df}=225)$, but not among controls $(r=-0.09, p=0.30, \mathrm{df}=128)$. Scatter plots and regression table are presented in Figure 2 and Table 2. In our prior paper examining change in anatomical measures as a function of conversion/non-conversion status, converters showed a steeper rate of expansion of the third ventricle compared with non-converters and controls, with a similar, though non-significant, tendency in the same direction with respect to lateral ventricular volume (Cannon et al., 2015).

\subsection{Relationship of subcortical and ventricular structures}

In order to examine whether ventricular enlargement is related to tissue loss in proximal subcortical structures, such as thalamus, caudate, pallidum, putamen, and hippocampus, we conducted independent tests for each structure to investigate whether changes in these brain structures are correlated with the change in ventricle size. However, none of the structures (all above $\mathrm{p}_{\mathrm{s}}>0.10$, corrected) were associated with expansion of ventricle size in either CHR cases or healthy controls or overall.

\subsection{Within Subject Replication}

A subset of the CHR individuals ( $n=125)$ and controls $(n=66)$ were followed for a 24-month FU (third time point) MRI assessment. Using the newly computed ARCH measures for the second interval (12-FU to 24-FU) the inverse relationship of ventricular expansion and cortical reduction among CHR individuals was replicated (see Figure 3 for scatter plots). Consistent with the results from the first interval, regression models for the second interval, predicting ARCH of mean cortical thickness and ARCH of SFC thickness, revealed significant group by ARCH ventricular volume interactions for both overall mean thickness $(t=3.12, p=0.0021)$ and SFC $(t=2.11, p=0.03)$. At a global level, ARCH of mean gray matter thickness was negatively correlated with ARCH of ventricular volume in the CHR group $(r=-0.41, p<0.0001, \mathrm{df}=118)$, but not in healthy controls $(r=0.12, p=0.34, \mathrm{df}=$ 61). Consistently, similar relationships were observed in SFC in the CHR group $(r=-0.28, p$ $=0.004, \mathrm{df}=118)$, while healthy controls did not show the same pattern $(\mathrm{r}=0.10, \mathrm{p}=0.43$, $\mathrm{df}=61)$. 


\section{Discussion}

The results of this study suggest that ventricular enlargement is linked in time to progressive reduction of gray matter in a broadly distributed set of cortical regions, rather than to focal subcortical tissue loss, among CHR youth. The cortical regions in which CHR individuals experiencing higher rates of ventricular expansion exhibited steeper rates of gray matter loss were primarily within heteromodal association cortices, including superior, medial, lateral, and inferior PFC, superior temporal gyrus, and parietal cortices. These findings suggest that the gray matter regions exhibiting aberrant rates of thinning in relation to psychosis risk are not limited to the regions in superior and medial PFC that survived the statistical threshold in our primary study (Cannon et al., 2015), but also extend to other cortical regions previously implicated in schizophrenia (Rimol et al., 2012). In contrast, the absence of significant association between ventricular volume and cortical thickness or subcortical volume at baseline suggests that the altered brain maturational pattern in CHR youth is an emergent process around the time of psychosis onset. It may be that such cross-sectional differences would not reliably reach statistical significance until the early stages of full-blown schizophrenia (i.e., first-episode psychosis).

That widespread regions of cortex may undergo thinning in relation to psychosis onset is consistent with the emerging consensus (Chung and Cannon, 2015), based on hundreds of neuroimaging studies of patients with schizophrenia (Pantelis, 2005; Shenton et al., 2001), that the pathophysiology of the disorder involves a network of multiple brain regions, rather than lesions in one or a few discrete areas (Stephan et al., 2009). In neuroimaging studies comparing a clinical group to healthy controls, the use of a mass-univariate analysis approach requires a rather severe correction of the observed p-values in light of the number of tests conducted. Application of such a threshold may limit identification of regions to those where the disease-related effects are maximal and may mask many other regions with moderate effects that are simply below the threshold (Davatzikos, 2004). In our primary study (Cannon et al., 2015), after FDR correction, regions showing significantly greater rates of reduction among CHR converters to psychosis were limited to superior and medial PFC. However, before correction, such regions included more inferior and lateral aspects of PFC, superior temporal gyrus, and parietal cortex; that is, coextensive with the topography of cortical regions showing significant reductions in association with ventricular enlargement.

In light of this pattern, and given the absence of relationships between ventricular enlargement and changes in subcortical volumes, expansion of ventricular spaces during the psychosis prodrome may be a consequence of this widespread, but subtle, thinning of the cortical ribbon. We conjecture that during the prodromal phase, cortical thinning results in ventricular expansion by reducing the mechanical pressures that extra-ventricular tissues exert against the ventricular wall, allowing the CSF-filled compartments to expand; in this sense, ventricular enlargement may represent a proxy for widespread cortical thinning during the psychosis prodrome. Although there is a slow and steady trajectory of shrinking cortex and ventricular expansion in healthy aging (Fjell et al., 2013; Raz, 2005), this was not detected in the healthy control group in this study, probably because it was relatively young (teens and twenties) and showed more restricted variability of anatomical changes within the one-year follow-up interval compared with the CHR group. Similarly, though we found no 
significant relationships between ventricular expansion and decreasing volumes of subcortical structures, and no differences at baseline or over time in volumes of subcortical structures between converters and non-converters (Cannon et al., 2015), it is possible that there are subtle changes in subcortical structures that we were not able to detect in the time frame and phase of illness that were under study. These findings, taken together with those of a prior study reporting an association between ventricular enlargement and shrinkage of thalamus and striatum in chronic schizophrenia (Gaser et al., 2004), suggest that atrophy of paraventricular structures may become a phenomenon especially or only after frank psychotic symptoms fully emerge. In any event, the null findings in the subcortical morphometric measures in CHR cases suggest that they are not the main culprit for influencing ventricular enlargement during the psychosis prodromal phase.

While the correlations noted in this study suggest that ventricular enlargement and cortical thinning occur in tandem (a finding that replicated across two one-year intervals), the design of this study does not allow us to draw firm conclusions regarding whether the ventricular enlargement is the consequence of the excessive gray matter loss. Future studies are encouraged to use multiple assessment waves with briefer intervals to determine whether changes in cortical thickness precede and predict changes in ventricular volume (as in timelagged analysis).

In summary, expansion of the ventricular spaces is linked in time with an accelerated rate of widespread cortical thinning prior to psychosis onset. The cortical regions experiencing altered maturation during the psychosis prodrome may be more widespread than the regionally specific clusters that have been identified in previous case-control studies (Borgwardt et al., 2008; Cannon et al., 2015; Pantelis et al., 2003). Clarifying the trajectories of different structural abnormalities associated with schizophrenia will help to identify the role that separate pathophysiological processes play in the development of the disorder.

\section{Supplementary Material}

Refer to Web version on PubMed Central for supplementary material.

\section{Acknowledgments}

The authors thank the following individuals for assistance with subject scheduling and/or scan acquisition: Angielette Andaya, Nurit Hirsh and Jamie Zinberg (UCLA); Richard Juelich (BIDMC-Harvard); M. Louis Lauzon, J. Stowkowy and C. Marshall (Calgary); Jason Nunag and Daniel Roman (UCSD); Nicole Popp Santamauro and Hedy Sarofin (Yale).

This work was supported by a collaborative U01 award from the National Institute of Mental Health at the National Institutes of Health (MH081902 to TDC; MH081857 to BAC; MH081988 to EW; MH081928 to LJS; MH082004 to DP; MH082022 to KC; MH081984 to JA; MH082022 to SWW) and NIMH P50 MH066286 and Staglin Music Festival for Mental Health (CEB), NIMH P50 MH080272, and the Commonwealth of Massachusetts (SCDMH82101008006) to L.J.S.

\section{References}

Addington J, Cadenhead KS, Cornblatt BA, Mathalon DH, McGlashan TH, Perkins DO, Seidman LJ, Tsuang MT, Walker EF, Woods SW, Addington JA, Cannon TD. North American Prodrome Longitudinal Study (NAPLS 2): overview and recruitment. Schizophrenia Research. 2012; 142:7782. [PubMed: 23043872] 
Borgwardt SJ, McGuire PK, Aston J, Gschwandtner U, Pflüger MO, Stieglitz RD, Radue EW, RiecherRössler A. Reductions in frontal, temporal and parietal volume associated with the onset of psychosis. Schizophrenia Research. 2008; 106:108-114. [PubMed: 18789654]

Cannon TD, Chung Y, He G, Sun D, Jacobson A, van Erp TGM, McEwen S, Addington J, Bearden CE, Cadenhead K, Cornblatt B, Mathalon DH, McGlashan T, Perkins D, Jeffries C, Seidman LJ, Tsuang M, Walker E, Woods SW, Heinssen R. North American Prodrome Longitudinal Study Consortium. Progressive reduction in cortical thickness as psychosis develops: a multisite longitudinal neuroimaging study of youth at elevated clinical risk. Biological Psychiatry. 2015; 77:147-157. [PubMed: 25034946]

Cannon TD, Sun F, McEwen SJ, Papademetris X, He G, van Erp TGM, Jacobson A, Bearden CE, Walker E, Hu X, Zhou L, Seidman LJ, Thermenos HW, Cornblatt B, Olvet DM, Perkins D, Belger A, Cadenhead K, Tsuang M, Mirzakhanian H, Addington J, Frayne R, Woods SW, McGlashan TH, Constable RT, Qiu M, Mathalon DH, Thompson P, Toga AW. Reliability of neuroanatomical measurements in a multisite longitudinal study of youth at risk for psychosis. Hum Brain Mapp. 2013; 35:2424-2434. [PubMed: 23982962]

Chung Y, Cannon TD. Brain imaging during the transition from psychosis prodrome to schizophrenia. The Journal of nervous and mental disease. 2015; 203:336-341. [PubMed: 25900551]

Chung Y, Jacobson A, He G, van Erp TGM, McEwen S, Addington J, Bearden CE, Cadenhead K, Cornblatt B, Mathalon DH, McGlashan T, Perkins D, Seidman LJ, Tsuang M, Walker E, Woods SW, Heinssen R, Cannon TD. Prodromal Symptom Severity Predicts Accelerated Gray Matter Reduction and Third Ventricle Expansion Among Clinically High Risk Youth Developing Psychotic Disorders. Mol Neuropsychiatry. 2015; 1:13-22. DOI: 10.1159/000371887 [PubMed: 26005673]

Dale AM, Fischl B, Sereno MI. Cortical surface-based analysis. I. Segmentation and surface reconstruction. NeuroImage. 1999; 9:179-194. DOI: 10.1006/nimg.1998.0395 [PubMed: 9931268]

Davatzikos C. Why voxel-based morphometric analysis should be used with great caution when characterizing group differences. NeuroImage. 2004; 23:17-20. DOI: 10.1016/j.neuroimage. 2004.05.010 [PubMed: 15325347]

DeLisi LE, Sakuma M, Maurizio AM, Relja M. Cerebral ventricular change over the first 10 years after the onset of schizophrenia. Psychiatry Res. 2004; 130:57-70. [PubMed: 14972368]

DeLisi LE, Sakuma M, Tew W, Kushner M, Hoff AL, Grimson R. Schizophrenia as a chronic active brain process: a study of progressive brain structural change subsequent to the onset of schizophrenia. Psychiatry Res. 1997; 74:129-140. [PubMed: 9255858]

Desikan RS, Ségonne F, Fischl B, Quinn BT, Dickerson BC, Blacker D, Buckner RL, Dale AM, Maguire RP, Hyman BT, Albert MS, Killiany RJ. An automated labeling system for subdividing the human cerebral cortex on MRI scans into gyral based regions of interest. NeuroImage. 2006; 31:968-980. DOI: 10.1016/j.neuroimage.2006.01.021 [PubMed: 16530430]

First, MB., Spitzer, RL., Gibbon, M., Williams, JB. Structured Clinical Interview for DSM-IV Axis I Disorders, Patient Edition. Vol. 1995. Research Department, New York State Psychiatric Institute; New York: 1995.

Fischl B, Dale AM. Measuring the thickness of the human cerebral cortex from magnetic resonance images. Proc Natl Acad Sci USA. 2000; 97:11050-11055. DOI: 10.1073/pnas.200033797 [PubMed: 10984517]

Fischl B, Salat DH, Busa E, Albert M, Dieterich M, Haselgrove C, van der Kouwe A, Killiany R, Kennedy D, Klaveness S. Whole brain segmentation: automated labeling of neuroanatomical structures in the human brain. Neuron. 2002; 33:341-355. [PubMed: 11832223]

Fischl B, Sereno MI, Dale AM. Cortical surface-based analysis: II: Inflation, flattening, and a surfacebased coordinate system. NeuroImage. 1999; 9:195-207. [PubMed: 9931269]

Fischl B, van der Kouwe A, Destrieux C, Halgren E, Ségonne F, Salat DH, Busa E, Seidman LJ, Goldstein J, Kennedy D, Caviness V, Makris N, Rosen B, Dale AM. Automatically parcellating the human cerebral cortex. Cereb Cortex. 2004; 14:11-22. DOI: 10.1093/cercor/bhg087 [PubMed: 14654453]

Fjell AM, Westlye LT, Grydeland H, Amlien I, Espeseth T, Reinvang I, Raz N, Holland D, Dale AM, Walhovd KB. Alzheimer Disease Neuroimaging Initiatives. Critical ages in the life course of the adult brain: nonlinear subcortical aging. Neurobiology of Aging. 2013; 34:2239-2247. [PubMed: 23643484] 
Gaser C, Nenadic I, Buchsbaum BR, Hazlett EA, Buchsbaum MS. Ventricular enlargement in schizophrenia related to volume reduction of the thalamus, striatum, and superior temporal cortex. Am J Psychiatry. 2004; 161:154-156. [PubMed: 14702264]

Genovese CR, Lazar NA, Nichols T. Thresholding of statistical maps in functional neuroimaging using the false discovery rate. NeuroImage. 2002; 15:870-878. [PubMed: 11906227]

Gogtay N, Giedd JN, Lusk L, Hayashi KM, Greenstein D, Vaituzis AC, Nugent TF, Herman DH, Clasen LS, Toga AW. Dynamic mapping of human cortical development during childhood through early adulthood. Proc Natl Acad Sci USA. 2004; 101:8174-8179. [PubMed: 15148381]

Huttenlocher PR. Synaptic density in human frontal cortex - developmental changes and effects of aging. Brain Res. 1979; 163:195-205. DOI: 10.1016/0006-8993(79)90349-4 [PubMed: 427544]

Huttenlocher PR, Dabholkar AS. Regional differences in synaptogenesis in human cerebral cortex. Journal of comparative Neurology. 1997; 387:167-178. [PubMed: 9336221]

McGlashan, T., Walsh, B., Woods, S. The Psychosis-Risk Syndrome: Handbook for Diagnosis and Follow-Up. Oxford University Press; 2010.

Pantelis C. Structural Brain Imaging Evidence for Multiple Pathological Processes at Different Stages of Brain Development in Schizophrenia. Schizophrenia Bulletin. 2005; 31:672-696. DOI: 10.1093/schbul/sbi034 [PubMed: 16020551]

Pantelis C, Velakoulis D, McGorry PD, Wood SJ, Suckling J, Phillips LJ, Yung AR, Bullmore ET, Brewer W, Soulsby B. Neuroanatomical abnormalities before and after onset of psychosis: a crosssectional and longitudinal MRI comparison. Lancet. 2003; 361:281-288. [PubMed: 12559861]

Raz N. Regional Brain Changes in Aging Healthy Adults: General Trends, Individual Differences and Modifiers. Cerebral Cortex. 2005; 15:1676-1689. DOI: 10.1093/cercor/bhi044 [PubMed: 15703252]

Reuter M, Fischl B. Avoiding asymmetry-induced bias in longitudinal image processing. NeuroImage. 2011; 57:19-21. DOI: 10.1016/j.neuroimage.2011.02.076 [PubMed: 21376812]

Reuter M, Rosas HD, Fischl B. Highly accurate inverse consistent registration: A robust approach. NeuroImage. 2010; 53:1181-1196. DOI: 10.1016/j.neuroimage.2010.07.020 [PubMed: 20637289]

Reuter M, Schmansky NJ, Rosas HD, Fischl B. Within-subject template estimation for unbiased longitudinal image analysis. NeuroImage. 2012; 61:1402-1418. DOI: 10.1016/j.neuroimage. 2012.02.084 [PubMed: 22430496]

Rimol LM, Nesvåg R, Hagler DJ, Bergmann O, Fennema-Notestine C, Hartberg CB, Haukvik UK, Lange E, Pung CJ, Server A, Melle I, Andreassen OA, Agartz I, Dale AM. Cortical volume, surface area, and thickness in schizophrenia and bipolar disorder. Biological Psychiatry. 2012; 71:552-560. DOI: 10.1016/j.biopsych.2011.11.026 [PubMed: 22281121]

Shenton ME, Dickey CC, Frumin M. A review of MRI findings in schizophrenia. Schizophrenia Research. 2001; 49:1-52.

Stephan KE, Friston KJ, Frith CD. Dysconnection in schizophrenia: from abnormal synaptic plasticity to failures of self-monitoring. Schizophrenia Bulletin. 2009; 35:509-527. DOI: 10.1093/schbul/ sbn176 [PubMed: 19155345]

Symonds LL, Archibald SL, Grant I, Zisook S, Jernigan TL. Does an increase in sulcal or ventricular fluid predict where brain tissue is lost? J Neuroimaging. 1999; 9:201-209. [PubMed: 10540599] 


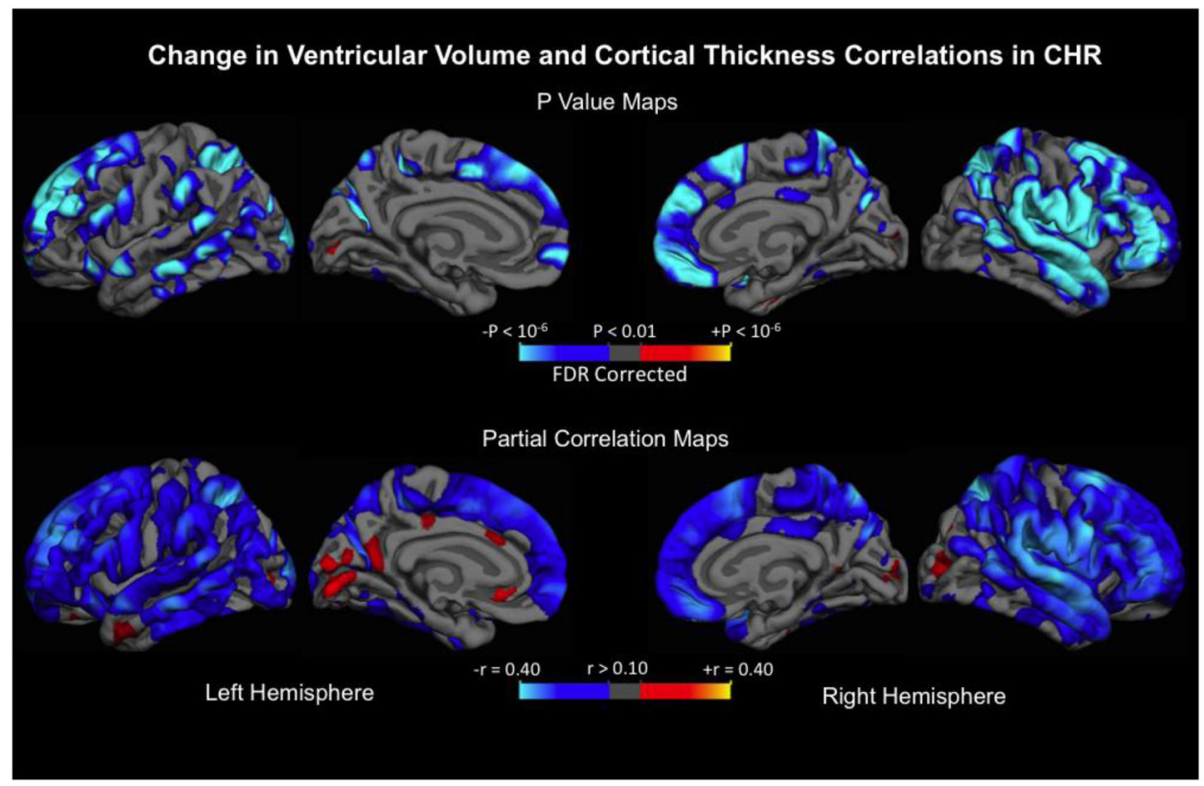

Figure 1.

Upper panel: Statistical p value maps showing regions where annualized rate of percent change (ARCH) of ventricle volume is associated with cortical gray matter thickness ARCH after controlling for age and sex in CHR sample $(\mathrm{n}=274)$. False discovery rate (FDR; 0.01) correction was applied for multiple comparisons. Regions associated with expanding ventricle and cortical thinning are represented in cooler color scheme. Lower panel: partial correlation coefficients after adjusting for age and sex are mapped across the surface. 


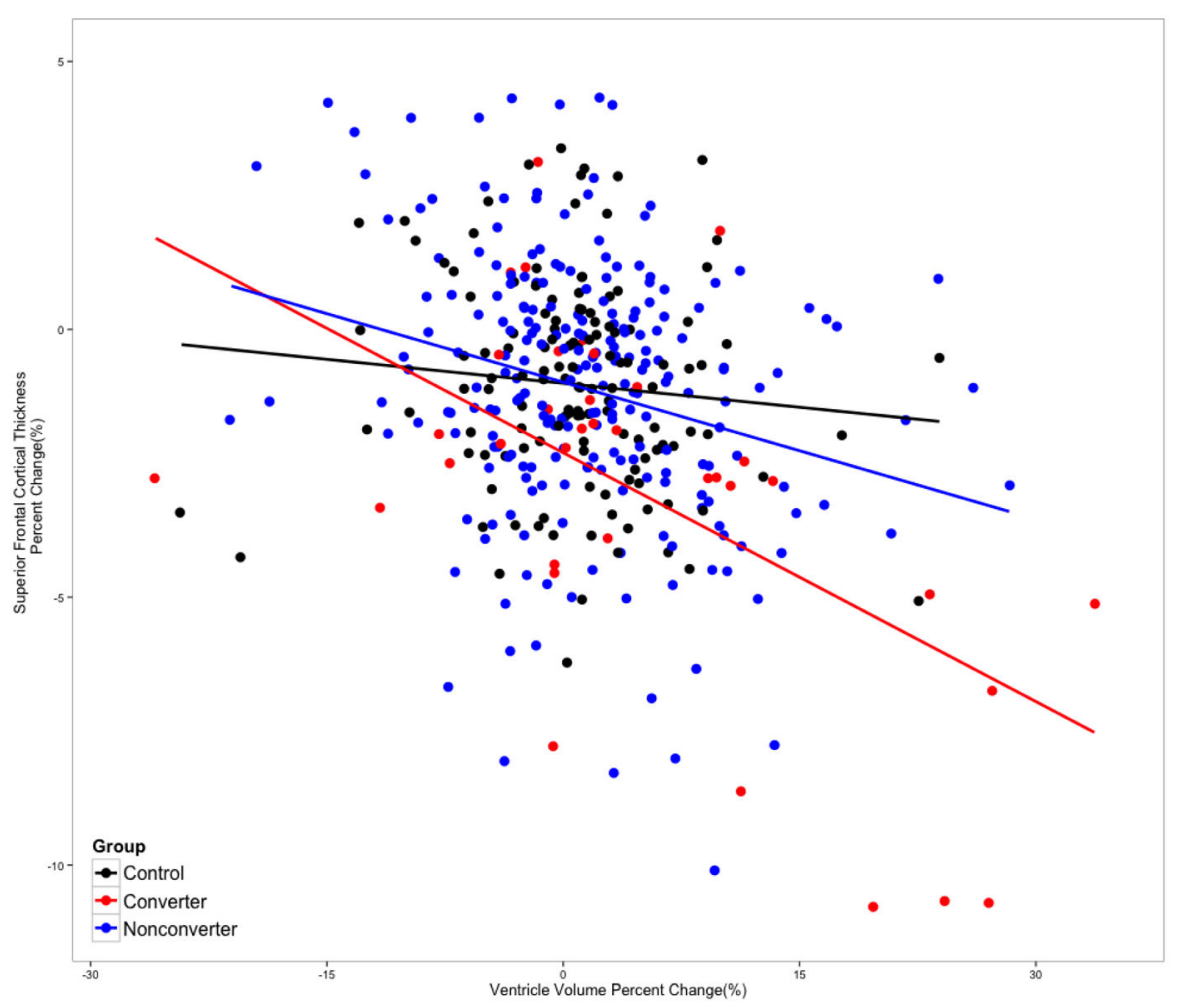

Figure 2.

Scatterplot for the association between annualized rate of percent change (ARCH) of ventricular volume and mean superior frontal cortical thickness by clinical group status. Baseline and second time point (12-FU) scans were used to calculate the ARCH measures. 

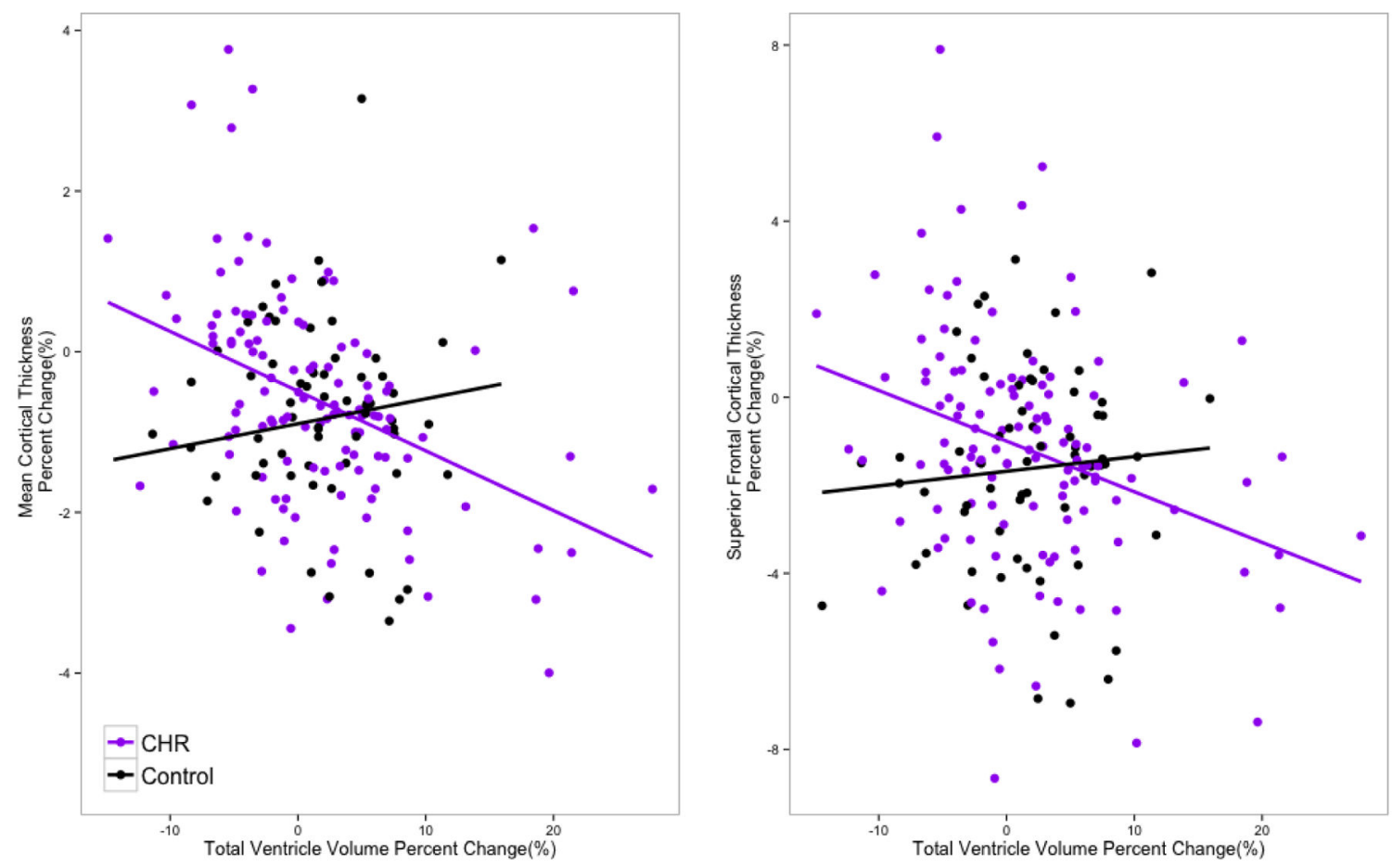

Figure 3.

Scatterplot for the annualized rate of percent change in ventricular volume with (Left) mean cortical thickness and (right) superior frontal cortical thickness between second (12-FU) and third (24-FU) time points among CHR $(n=121)$ and healthy control subjects $(n=64)$. 


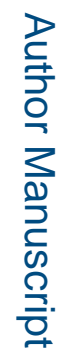

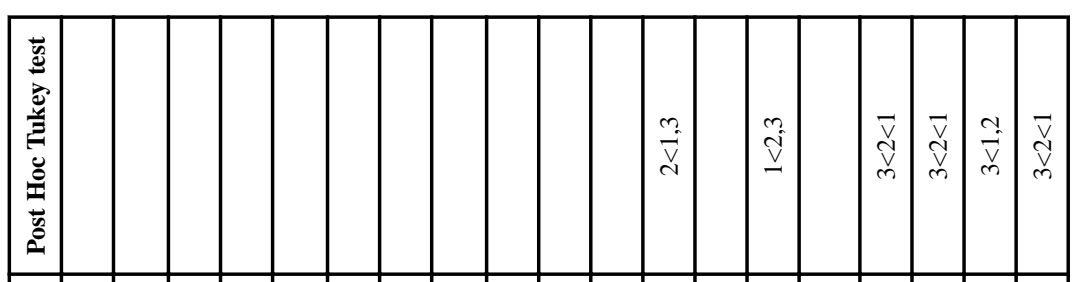

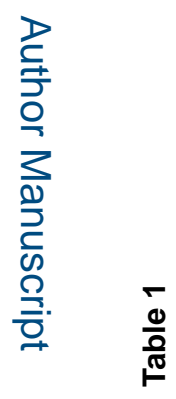

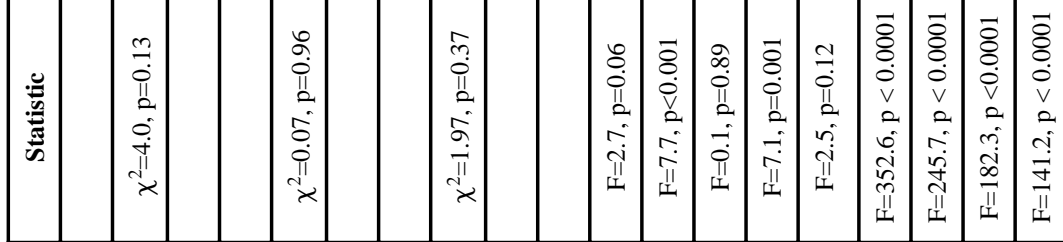

I

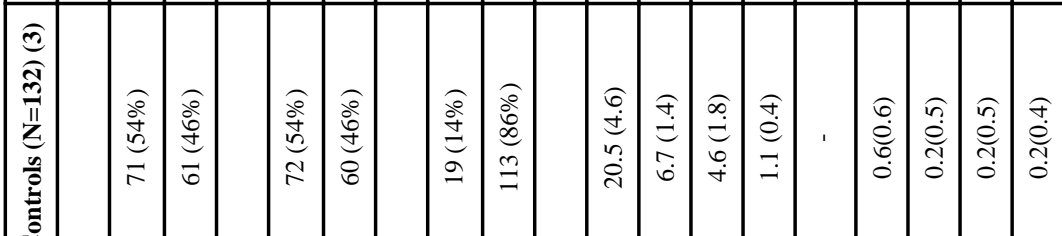

ב⿳亠口冋亍

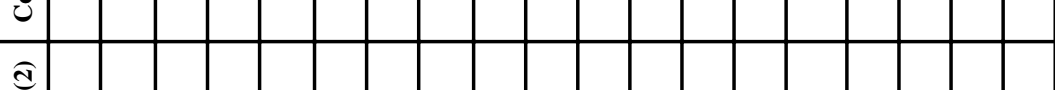

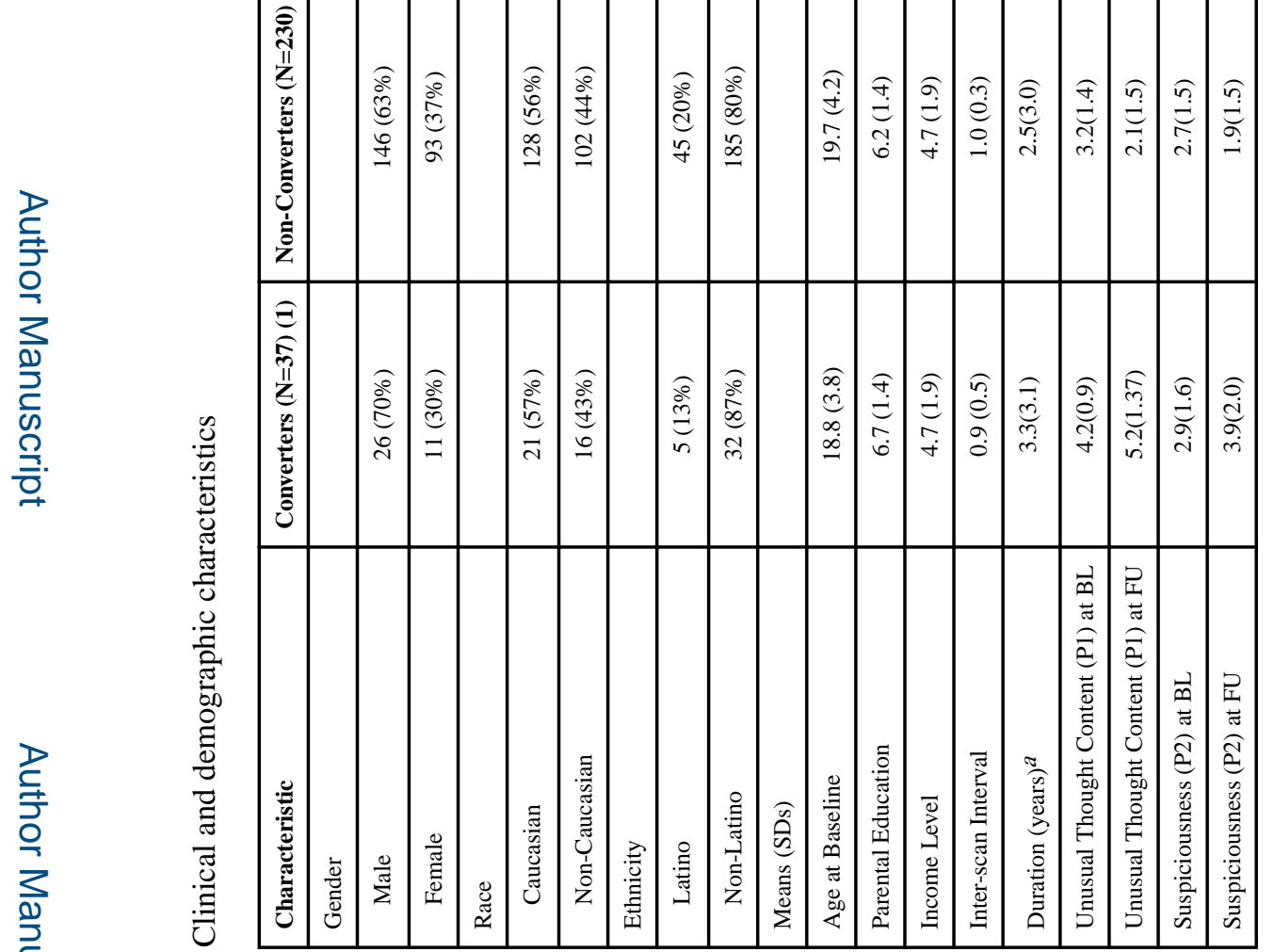

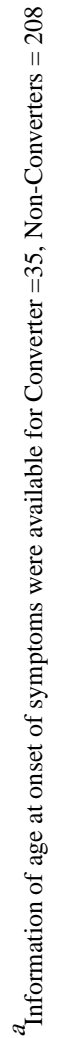

Schizophr Res. Author manuscript; available in PMC 2018 November 01. 
Table 2

Regression model predicting ARCH Superior Frontal Cortical thickness ( $\mathrm{N}=395)$

\begin{tabular}{lllll}
\hline \multicolumn{5}{c}{ Mean ARCH SFC Thickness } \\
\hline Term & $\boldsymbol{B}$ & SE & $\mathbf{t}$ & $\boldsymbol{P}$ (two-tailed) \\
\hline Sex & $1.44 \mathrm{e}-3$ & $2.33 \mathrm{e}-3$ & 0.62 & 0.54 \\
Age & $1.87 \mathrm{e}-4$ & $2.65 \mathrm{e}-4$ & 0.71 & 0.48 \\
ARCH Ventricular Volume & -0.023 & 0.030 & -0.76 & 0.45 \\
Converter & $\mathbf{- 0 . 0 1 1}$ & $\mathbf{4 . 5 e}-\mathbf{3}$ & $\mathbf{- 2 . 3 8}$ & $\mathbf{0 . 0 1 8}$ \\
Non-Converter & $1.731-4$ & $2.5 \mathrm{e}-3$ & 0.069 & 0.94 \\
ARCH Ventricular Volume x Converter & $\mathbf{- 0 . 1 2}$ & $\mathbf{0 . 0 4 4}$ & $\mathbf{- 2 . 8 3}$ & $\mathbf{0 . 0 0 4 9}$ \\
ARCH Ventricular Volume x Non-Converter & -0.051 & 0.037 & -1.40 & 0.16 \\
\hline
\end{tabular}

Model Controlled for Scanner; ARCH: Annualized Rate of Change; SFC: Superior Frontal Cortex 